\title{
RINGS CHARACTERIZED BY THEIR RIGHT IDEALS OR CYCLIC MODULES
}

\author{
by DINH VAN HUYNH*, NGUYEN V. DUNG and PATRICK F. SMITH
}

(Received 10th August 1987)

\begin{abstract}
It is well known that a ring $R$ is semiprime Artinian if and only if every right ideal is an injective right $R$-module. In this paper we shall be concerned with the following general question: given a ring $R$ all of whose right ideals have a certain property, what implications does this have for the ring $R$ itself? In practice, it is not necessary to insist that all right ideals have the property, usually the maximal or essential right ideals will suffice. On the other hand, Osofsky proved that a ring $R$ is semiprime Artinian if and only if every cyclic right $R$-module is injective. This leads to the second general question: given a ring $R$ all of whose cyclic right $R$-modules have a certain property, what can one say about $R$ itself?
\end{abstract}

\section{Right artinian rings}

All rings considered are associative with identity and all modiles are unitary. Let $R$ be a ring and $M$ a right $R$-module. The socle of $M$ will be denoted soc $M$. The module $M$ is called a max module if every proper submodule is contained in a maximal submodule. For example, a well known consequence of Zorn's Lemma is that finitely generated $R$ modules are max modules. On the other hand, semisimple $R$-modules are max modules. To be more precise we have:

Lemma 1.1. Let $R$ be a ring. $A$ right $R$-module $M$ is semisimple if and only if $M$ is a max module which does not contain an essential maximal submodule.

Proof. The necessity is proved by [1, Theorem 9.6]. Conversely, suppose that $M$ is a max module which does not contain an essential maximal submodule. Suppose that $M \neq \operatorname{soc} M$. Then there exists a maximal submodule $K$ of $M$ such that $\operatorname{soc} M \subseteq K$. By hypothesis $K$ is not essential and hence there exists a non-zero submodule $U$ of $M$ such that $K \cap U=0$. Clearly $U$ is simple and hence $U \subseteq \operatorname{soc} M \subseteq K$, a contradiction. Thus $M=\operatorname{soc} M$ and $M$ is semisimple.

Semiprime Artinian rings are characterized either by the fact that every maximal right

* Research supported by the Alexander von Humboldt-Stiftung. 
ideal is injective or by the fact that every essential right ideal is injective (see [1, Theorem 9.6 and Proposition 13.9]). A generalization of this result is a consequence of the following theorem.

Theorem 1.2. Let $R$ be a ring and $M$ a finitely generated right $R$-module. Then $M$ is Artinian (respectively, Noetherian) if and only if every essential maximal submodule of $M$ is the direct sum of an injective module and an Artinian (respectively, Noetherian) module.

Proof. We consider the Artinian case. The Noetherian case is proved similarly. The necessity is trivial. Conversely, suppose that every essential maximal submodule of $M$ is the direct sum of an injective module and an Artinian module. Let $A$ denote the sum of all Artinian submodules of $M$. Suppose that $A \neq M$. Then there exists a maximal submodule $K$ of $M$ such that $A \subseteq K$. If $K$ is not essential then there exists a non-zero submodule $U$ of $M$ such that $K \cap U=0$ and in this case $U$ is simple and hence $U \subseteq A \subseteq K$, giving $U=0$, a contradiction. Thus $K$ is essential. By hypothesis there exist an injective submodule $I$ and an Artinian submodule $B$ such that $K=I \oplus B$. But $K / B \cong I$ implies that $K / B$ is an injective submodule of $M / B$ and hence there exists a submodule $C$ of $M$ containing $B$ such that $M / B=(K / B) \oplus(C / B)$. Note that $C / B \cong M / K$ and hence $C / B$ is simple and $C$ is Artinian. Thus $C \subseteq A$ and $M=K+C \subseteq K$, a contradiction. Therefore $A=M$. Since $M$ is finitely generated it follows that $M=A_{1}+\cdots+A_{n}$ for some positive integer $n$ and Artinian submodules $A_{i}(1 \leqq i \leqq n)$. Thus $M$ is Artinian, as required.

The same proof not only applies to Noetherian modules but also to finitely generated modules of Krull dimension $\leqq \alpha$, for any given ordinal $\alpha \geqq 0$. Theorem 1.2 has the following immediate consequence.

Corollary 1.3. A ring $R$ is right Artinian (respectively, right Noetherian) if and only if every essential maximal right ideal of $R$ is the direct sum of an injective right ideal and an Artinian (respectively, Noetherian) right ideal.

We shall apply Corollary 1.3 to hereditarily Artinian rings. Let $R$ be a right Artinian ring. In general, a (two-sided) ideal of $R$ will not be a right Artinian ring. The ring $R$ is called hereditarily Artinian provided every ideal of $R$ is a right Artinian ring. Such rings have been discussed in various papers and several different characterizations have been given (see, for example, $[3,7,9,10])$. In particular, by $[7$, Hauptsatz] we know that a ring $R$ is hereditarily Artinian if and only if $R$ is the direct sum of a semiprime Artinian ring and a finite ring. We shall characterize these rings in terms of their right ideals, and to do so we first prove:

Lemma 1.4. Let $A \subseteq B$ be right ideals of a right Artinian ring $R$ such that $A$ is an essential submodule of $B$ and $A$ is finite. Then $B$ is finite.

Proof. By [2, Theorem 2], $R$ contains an ideal $R^{*}$ such that $R / R^{*}$ is finite and $R^{*}$ 
contains no non-zero finite right ideals. Thus $A \cap R^{*}=0$ and hence, because $A$ is essential in $B, B \cap R^{*}=0$. Thus $B$ embeds in $R / R^{*}$ and $B$ is finite.

Theorem 1.5. A ring $R$ is hereditarily Artinian if and only if every essential maximal right ideal is the direct sum of an injective right ideal and a finite right ideal.

Proof. The necessity follows by [7, Hauptsatz]. Conversely, suppose that every essential maximal right ideal is the direct sum of an injective right ideal and a finite right ideal. By Corollary 1.3 the ring $R$ is right Artinian. Let $F$ be the sum of all the finite right ideals of $R$. Note that $F$ is an ideal of $R$. Since $R$ is right Noetherian it follows that $F$ is finite. Let $M / F$ be any essential maximal right ideal of $R / F$. Then $M$ is an essential maximal right ideal of $R$ and by hypothesis there exist an injective right ideal $I$ and a finite right ideal $G$ such that $M=I \oplus G$. Since $M / G \cong I$ it follows that $M / G$ is injective and hence there exists a right ideal $H$ containing $G$ such that $R / G=$ $(M / G) \oplus(H / G)$. Now $M \cap H=G$ so that $M \cap H$ is finite. Moreover, $M \cap H$ is an essential submodule of $H$. By Lemma 1.4 it follows that $H$ is finite and hence $H \subseteq F$. Thus $R=M+H \subseteq M$, a contradiction. We have proved that the ring $R / F$ has no essential maximal right ideals and hence, by Lemma $1.1, R / F$ is semiprime. If $J$ is the Jacobson radical of $R$ then $J \subseteq F$, i.e. $J$ is finite. By [4, Theorem 1] $R$ is hereditarily Artinian.

\section{Serial rings}

It is easy to give examples of rings which have the property that every proper right ideal is the direct sum of an injective right ideal and a semisimple right ideal, but which are not right Artinian (or right Noetherian). For example. let $K$ be any field, $V$ any infinite dimensional vector space over $K$ and $R$ the ring of all $2 \times 2$ "matrices" of the form

$$
\left[\begin{array}{ll}
k & v \\
0 & k
\end{array}\right]
$$

where $k \in K, v \in V$. Then $R$ is a commutative ring with a unique maximal ideal $M$ consisting of all matrices of the above form with $k=0$. Clearly any proper ideal of $R$ is semisimple but $R$ is neither Artinian nor Noetherian. Note that as an $R$-module the socle of $R$ is the unique maximal submodule of $R$. The first result of this section provides more examples of modules with the property that every submodule is the direct sum of an injective module and a semisimple module.

Lemma 2.1. Let $R$ be $a$ ring and $M$ a projective right $R$-module such that $M=$ $E_{1} \oplus \cdots \oplus E_{n}$, where $n$ is a positive integer and $E_{i}$ is either semisimple or injective of (composition) length 2 for each $1 \leqq i \leqq n$. Then every submodule of $M$ is the direct sum of an injective module and a semisimple module.

Proof. Note first that the module $M /(\operatorname{soc} M)$ has finite length. Let $N$ be a submodule 
of $M$. The proof is by induction on the length of $N /(\operatorname{soc} N)$. Let $U_{i}=\operatorname{soc} E_{i}(1 \leqq i \leqq n)$. Let $\pi_{i}: M \rightarrow E_{i}(1 \leqq i \leqq n)$ denote the canonical projection maps. If $\pi_{i}(N) \subseteq U_{i}(1 \leqq i \leqq n)$ then $N$ is semisimple. Suppose that there exists $1 \leqq j \leqq n$ such that $\pi_{j}(N) \nsubseteq U_{j}$. Since $E_{j}$ has length 2 it follows that $\pi_{j}(N)=E_{j}$. But $E_{j}$ is projective, being a direct summand of $M$, and hence there exist submodules $N_{i}(i=1,2)$ such that $N_{1} \cong E_{j}$ and $N=N_{1} \oplus N_{2}$. Note that $N /(\operatorname{soc} N) \cong\left(N_{1} /\left(\operatorname{soc} N_{1}\right)\right) \oplus\left(N_{2} /\left(\operatorname{soc} N_{2}\right)\right)$ and $N_{1} \neq \operatorname{soc} N_{1}$. By induction on the length of $N /(\operatorname{soc} N)$, the submodule $N_{2}$, and hence $N$ itself, is the direct sum of an injective module and a semisimple module.

Before proving the next result we make a simple observation about max modules. Let $R$ be a ring and $M$ a right $R$-module such that there exists a finite chain

$$
M=M_{0} \supseteq M_{1} \supseteq \cdots \supseteq M_{k}=0,
$$

of submodules $M_{i}(0 \leqq i \leqq k)$ of $M$ such that $M_{i-1} / M_{i}$ is semisimple for each $1 \leqq i \leqq k$. Then $M$ is a max module. Let $S=M_{k-1}$. If $N$ is a proper submodule of $M$ then consider the submodule $N+S$. If $N+S \neq M$ then $(N+S) / S$ is a proper submodule of $M / S$ so that by induction $(N+S) / S$ is contained in a maximal submodule of $M / S$. Thus $N+S$, and hence $N$, is contained in a maximal submodule of $M$. On the other hand if $M=N+S$ then $N \cap S$ is a submodule of the semisimple module $S$ so that by [1, Theorem 9.6], there exists a submodule $K$ of $S$ such that $S=(N \cap S) \oplus K$. It follows that $M=N \oplus K$. Clearly $K \neq 0$ and if $L$ is a maximal submodule of $K$ then $N \oplus L$ is a maximal submodule of $M$ containing $N$. Thus $M$ is a max module. As a partial converse to Lemma 2.1 we have the following result.

Lemma 2.2. Let $R$ be $a$ ring and $M$ a max right $R$-module such that every essential maximal submodule of $M$ is the direct sum of an injective module and a semisimple module. Then $M /(\operatorname{soc} M)$ is semisimple and $M$ is the direct sum $I \oplus A$ of an injective module $I$ and a module $A$ which contains a semisimple maximal submodule.

Proof. Let $S=\operatorname{soc} M$ and let $T$ be the submodule of $M$ containing $S$ such that $T / S=\operatorname{soc}(M / S)$. Suppose that $T \neq M$. Then there exists a maximal submodule $K$ of $M$ such that $T \subseteq K$. It is clear that $K$ is essential in $M$. By hypothesis there exist an injective submodule $I$ and a semisimple submodule $B$ such that $K=I \oplus B$. Since $K / B$ is injective, being isomorphic to $I$, there exists a submodule $A$ containing $B$ such that

$$
M / B=(K / B) \oplus(A / B) .
$$

But $A / B$ is simple, being isomorphic to $M / K$, and hence $A \subseteq S$ or $A \cap S=B$. Thus in any case $A$ contains a semisimple maximal submodule. Note further that $M=I \oplus A$. Moreover $(A+S) / S \cong A /(A \cap S)$ and hence $(A+S) / S$ is zero or simple. In any case $A \subseteq T \subseteq K$ and $M=K+A=K$, a contradiction. Thus $T=M$ and $M / S$ is semisimple.

Lemma 2.3. Let $R$ be $a$ ring and $M$ a finitely generated right $R$-module such that 
every essential maximal submodule is the direct sum of an injective module and a finitely generated semisimple module. Then $M$ contains a semisimple maximal submodule or $M$ is a finite direct sum of simple modules and injective modules of length 2. The converse holds if $M$ is projective.

Proof. By Theorem 1.2 M has finite length and by Lemma $2.2 \mathrm{M}$ is a direct sum $I \oplus A$ of an injective module $I$ and a module $A$ which contains a semisimple maximal submodule. Suppose $I \neq 0$. Since $M$ has finite length there exists a positive integer $n$ and indecomposable injective submodules $E_{i}(1 \leqq i \leqq n)$ such that $M=E_{1} \oplus \cdots \oplus E_{n} \oplus A$. If $E_{i}$ is simple for each $1 \leqq i \leqq n$ then $M$ contains a semisimple maximal submodule. Suppose that $E_{1}$ is not simple. Let $N$ be a maximal submodule of $E_{1}$. Then $N \oplus E_{2} \oplus \cdots \oplus E_{n} \oplus A$ is an essential maximal submodule of $M$ and thus, by hypothesis, is a finite direct sum of indecomposable injective modules and simple modules. By the Krull-Schmidt Theorem (see [1, Theorem 12.9]) $N$ and $A$ are both finite direct sums of indecomposable injective submodules and simple submodules. Since $N$ is uniform it follows that $N$ is simple and hence $E_{1}$ has length 2 . It follows that $M$ is a finite direct sum of simple modules and injective modules of length 2 .

We shall call a module $M$ almost semisimple provided $\operatorname{soc} M$ is the unique maximal submodule of $M$. Note that any finitely generated almost semisimple module is cyclic. For, if $M$ is finitely generated and almost semisimple then there exists $x \in M$ such that $x \notin \operatorname{soc} M$. Then $x R$ is not contained in a maximal submodule of $M$ and hence $M=x R$.

Theorem 2.4. Let $R$ be a ring and $M$ a finitely generated right $R$-module. Consider the statements:

(i) every proper submodule of $M$ is the direct sum of an injective module and a finitely generated semisimple module;

(ii) every maximal submodule of $M$ is the direct sum of an injective module and a finitely generated semisimple module;

(iii) $M$ has finite length and $M$ is almost semisimple or $M$ is a finite direct sum of simple modules and injective modules of length 2.

Then $(i) \Rightarrow(i i) \Rightarrow($ iii $)$. Moreover if $M$ is projective then $($ iii $) \Rightarrow(i)$.

Proof. (i) $\Rightarrow$ (ii). Obvious.

(ii) $\Rightarrow$ (iii). Suppose (ii) holds. Then $M$ has finite length by Theorem 1.2. By Lemma 2.3 we can suppose without loss of generality that $\operatorname{soc} M$ is a maximal submodule of $M$. If $M$ is not almost semisimple then $M$ contains a maximal submodule $K \neq \operatorname{soc} M$. By hypothesis there exist an injective submodule $I$ and a semisimple submodule $A$ such that $K=I \oplus A$. Clearly $I \neq 0$ and hence $M$ contains a submodule $E$ which is not simple but $E$ is the injective envelope of a simple submodule $U$ of $M$. Now $M=E \oplus B$, for some submodule $B$ of $M$, and 


$$
M /(\operatorname{soc} M) \cong(E / U) \oplus(B /(\operatorname{soc} B))
$$

implies that $E / U$ is simple and $B$ is semisimple. Thus $E$ has length 2 and $M$ is a finite direct sum of $E$ and simple submodules. This proves (iii).

Now suppose that $M$ is projective and $M$ is a finite direct sum of simple modules and injective modules of length 2. By Lemma 2.1 every submodule of $M$ is the direct sum of an injective module and a semisimple module. On the other hand if $M$ is almost semisimple then every proper submodule is semisimple.

Corollary 2.5. The following statements are equivalent for a ring $R$.

(i) Every proper right ideal is the direct sum of an injective module and a finitely generated semisimple right ideal.

(ii) Every maximal right ideal is the direct sum of an injective module and a finitely generated semisimple right ideal.

(iii) The right $R$-module $R$ is almost semisimple or $R$ is a finite direct sum of minimal right ideals and injective right ideals of length 2 .

In Corollary 2.5 , if $R$ is a ring which satisfies (ii) then it does not follow that every right ideal of $R$ is the direct sum of an injective module and a finitely generated semisimple right ideal. We can provide an easy counter example. Let $K$ be any field, $V$ any $K$-vector space of finite dimension $\geqq 2$ and $S$ the ring of all "matrices"

$$
\left[\begin{array}{ll}
k & v \\
0 & k
\end{array}\right]
$$

where $k \in K$ and $v \in V$. It is clear that $S$ is a commutative Artinian ring whose socle is the unique maximal ideal which consists of all matrices of the above form with $k=0$. However the $S$-module $S$ is indecomposable and is neither injective nor simple. Further note that if any of the conditions of Corollary 2.5 hold then, by Lemma 2.1, every proper essential right ideal is the direct sum of an injective module and a finitely generated semisimple right ideal. The converse is false as the following example shows. Let $R=S \oplus K$, where $S$ and $K$ are as before. Again $R$ is a commutative Artinian ring whose socle is a maximal ideal. Thus every proper essential ideal of $R$ is semisimple. However the ideal $I=S \oplus 0$ is indecomposable and is neither injective nor simple. Thus not every submodule of $R_{R}$ is the direct sum of an injective module and a semisimple module.

Osofsky [8] proved that a ring $R$ is semiprime Artinian if and only if every cyclic right $R$-module is injective. A module $M$ is finitely cogenerated provided it has finitely generated essential socle. Artinian modules are finitely cogenerated. In [4] van Huynh and Dung proved that a ring $R$ is right Artinian if and only if every cyclic right $R$ module is the direct sum of an injective module and a finitely cogenerated module. Also van Huynh [3] proved that a ring $R$ is hereditarily Antinian if and only if every cyclic right $R$-module is the direct sum of an injective module and a finite module. 
A module $M$ is called uniserial if its set of submodules is linearly ordered. A ring $R$ is defined to be right serial if $R$ is a direct sum of uniserial right ideals. Left serial rings are defined similarly. By an Artinian serial ring we shall mean a ring which is right and left Artinian and right and left serial.

Theorem 2.6. The following statements are equivalent for a ring $R$ with Jacobson radical $J$.

(i) Every right ideal is the direct sum of an injective module and a finitely generated semisimple right ideal.

(ii) Every essential right ideal is the direct sum of an injective module and a finitely generated semisimple right ideal.

(iii) $R$ is a direct sum of minimal right ideals and injective right ideals of length 2.

(iv) Every cyclic right $R$-module is the direct sum of an injective module and a semisimple module.

(v) Every right $R$-module is the direct sum of an injective module and a semisimple module.

(vi) $R$ is an Artinian serial ring such that $J^{2}=0$.

(vii) Any of the left sided analogues of $(i)-(v)$.

Proof. (iii) $\Rightarrow$ (ii). By Lemma 2.1 .

(ii) $\Rightarrow$ (i). Suppose (ii) holds. By Corollary $1.3 R$ is right Artinian. Any right ideal is a direct summand of an essential right ideal so that (i) holds by the Krull-Schmidt Theorem.

(i) $\Rightarrow$ (iii). Suppose that the right $R$-module $R$ is almost semisimple. Then $R_{R}$ is indecomposable so that $R_{R}$ is injective or simple. If $R_{R}$ is injective then, being indecomposable and almost semisimple, $R_{R}$ has length 2. By Corollary 2.5, (iii) follows.

(iii) $\Rightarrow$ (iv). Suppose that there exists a positive integer $n$ such that $R=A_{1} \oplus \cdots \oplus A_{n}$ where $A_{i}$ is a minimal right ideal or an injective right ideal of length 2 for each $1 \leqq i \leqq n$. Let $X=x R$ be a cyclic right $R$-module. Note that $R$ is right Artinian and $X$ has finite length. Further note $X=x A_{1}+\cdots+x A_{n}$. Suppose that $X$ is not semisimple. Then $x A_{i}$ is not semisimple for some $1 \leqq i \leqq n$. Define $\phi: A_{i} \rightarrow x A_{i}$ by $\phi(a)=x a\left(a \in A_{i}\right)$. Then $\phi$ is an $R$-epimorphism. Since $x A_{i}$ is not semisimple it follows that $A_{i}$ is injective of length 2 and ker $\phi=0$. Thus $x A_{i}$, and hence $X$, contains an injective submodule $\operatorname{im} \phi$. There exists a submodule $Y$ of $X$ such that $X=(\operatorname{im} \phi) \oplus Y$. Note that $Y$ is cyclic and by induction on the length of $X$ it follows that $Y$, and hence $X$, is a direct sum of an injective module and a semisimple module. This proves (iv).

(iv) $\Rightarrow$ (v). By [4, Theorem 1.1] $R$ is right Artinian. Let $M$ be a right $R$-module. Because $R$ is right Noetherian, the union of any chain of injective submodules of $M$ is injective. By Zorn's Lemma $M$ contains a maximal injective submodule $I$. There exists a submodule $N$ of $M$ such that $M=I \oplus N$. Let $x \in N$. By hypothesis there exist an 
injective module $K$ and a semisimple module $S$ such that $x R=K \oplus S$. By the choice of $I$ it follows that $K=0$ and hence $x R=S$. Thus $N$ is semisimple. This proves (v).

(v) $\Rightarrow$ (vi). Suppose (v) holds. By (i) $\Rightarrow$ (iii) we know that $R$ is right Artinian right serial and $J^{2}=0$. Let $E$ be an indecomposable injective right $R$-module. Suppose that $E$ is not simple. Then $E$ is not semisimple and hence there exists $y \in E$ such that $y R$ is not semisimple. By (v) $y R$ is injective and hence $E=y R$. If $K$ is a maximal submodule of $E$ then $K$ is not injective and hence $K$ is simple by (ii). Thus $E$ is simple or $E$ has length 2 . By (v) and [1, Theorem 25.6] it follows that every right $R$-module is a direct sum of uniserial right $R$-modules. By [5, Theorem 1.3] $R$ is an Artinian serial ring.

(vi) $\Rightarrow$ (i). Suppose that $R$ is not semiprime Artinian. Let $A$ be a right ideal of $R$ which is indecomposable but is not simple. Let $H$ denote the injective envelope of $A$. Then $H$ is indecomposable and by [6, Theorem 25.4.2] $H$ is cyclic and uniserial, say $H=h R$. Now $R=B_{1} \oplus \cdots \oplus B_{k}$ for some positive integer $k$ and right ideals $B_{i}$ of length $\leqq 2$ for each $1 \leqq i \leqq k$. Now $H=h B_{1}+\cdots+h B_{k}$ implies $H=h B_{j}$ for some $1 \leqq j \leqq k$ (because $H$ is uniserial). Thus $H$ is a homomorphic image of $B_{j}$ and $H$ has length $\leqq 2$. It follows that $A=H$. Thus every right ideal is the direct sum of an injective module and a finitely generated semisimple right ideal.

We have proved the equivalence of (i)-(vi). That (vii) is also equivalent to the other conditions follows from the symmetry of (vi).

\section{REFERENCES}

1. F. W. Anderson and K. W. Fuller, Rings and Categories of Modules (Springer-Verlag, 1974).

2. Dinh van Huynh, A note on Artinian rings, Arch. Math. (Basel), 33 (1979), 546-553.

3. Dinh van Huynh, Some characterizations of hereditarily Artinian rings, Glasgow Math. J. 28 (1986), 21-23.

4. Dinh van Huynh and Nguyen V. Dung, A characterization of Artinian rings, Glasgow Math. J. 30 (1988), 67-73.

5. D. Eisenbud and P. Griffith, The structure of serial rings, Pacific J. Math. 36 (1971), 109-121.

6. C. FAITH, Algebra II: Ring Theory (Springer-Verlag, 1976).

7. A. Kertesz and A. Widiger, Artinsche Ringe mit artinshem Radikal, J. Reine Angew, Math. $242(1970), 8-15$.

8. B. L. Osofsky, Non-injective cyclic modules, Proc. Amer. Math. Soc. 19 (1968), 1383-1384.

9. A. WIDIGER, Lattice of radicals for hereditarily Artinian rings, Math. Nachr. 84 (1978), 301-309.

10. A. Widiger and R. WIEgand, Theory of radicals for hereditarily Artinian rings, Acta Sc. Math. Szeged 39 (1977), 303-312.

Institute of Mathematics

P.O. Box 631 Bo Ho

Hanol. Vietnam
Department of Mathematics

UNIVERSITY OF GLASGOW

Glasgow G12 8QW, Scotland, U.K. 